\title{
Proton Magnetic Resonance Spectroscopy and Magnetoencephalographic Estimation of Delta Dipole Density: A Combination of Techniques That May Contribute to the Diagnosis of Alzheimer's Disease
}

\author{
Alberto Fernández $^{a}$ Juan Manuel García-Segura ${ }^{b, c}$ Tomás Ortiz ${ }^{a}$ Julia Montoya ${ }^{b}$ \\ Fernando Maestú $^{a}$ Pedro Gil-Gregorio ${ }^{d}$ Pablo Campo $^{a}$ Juan Viaño ${ }^{b}$ \\ ${ }^{a}$ Centro de Magnetoencefalografía Dr. Pérez-Modrego, Universidad Complutense de Madrid, Madrid, \\ ${ }^{\mathrm{b}}$ Unidad de Resonancia Magnética, Clínica Ntra. Sra. del Rosario, Madrid, ' ${ }^{\mathrm{D}}$ Departamento de Bioquímica y \\ Biología Molecular, Facultad de Biología, Universidad Complutense de Madrid, Madrid, ' Servicio de Geriatría, \\ Hospital Universitario San Carlos de Madrid, Madrid, Spain
}

\section{Key Words}

Magnetoencephalography · Proton magnetic resonance spectroscopy · Alzheimer's disease $\cdot$

Myoinositol/N-acetyl aspartate $(\mathrm{ml} / \mathrm{NAA}) \cdot$ Delta dipole density

\begin{abstract}
Whole-head magnetoencephalographic recordings were obtained from 10 patients with Alzheimer's disease (AD) and 10 healthy controls in a resting position. Spectroscopic examinations were performed by means of a 1.5tesla whole-body scanner in the temporoparietal regions of both hemispheres. The relationship between ${ }^{1} \mathrm{H}$-MRSbased and magnetoencephalography (MEG)-based measures and their conjoined capability to improve the diagnosis of $A D$ were investigated in this study. Logistic regression analyses were performed. Three separated logistic models were calculated for ${ }^{1} \mathrm{H}$-MRS-based metabolites, low-frequency magnetic activity, and the combination of both measures. A combined myoinositol/Nacetyl aspartate (ml/NAA)-delta dipole density (DD) model predicted the diagnosis with $90 \%$ sensitivity and $100 \%$ specificity. Additionally, the combination of temporoparietal ml/NAA and delta DD values explained the
\end{abstract}

variability of individuals' cognitive status. The results support the notion that a multidisciplinary approach may improve the understanding and diagnosis of $A D$.

Copyright (C) 2005 S. Karger AG, Basel

\section{Introduction}

Alzheimer's disease (AD) is a degenerative disorder which causes neuronal loss and progressive cognitive deterioration. It is considered the major cause of dementia and its complex and multifactorial nature is broadly accepted. Additionally, there are no clear-cut positive indicators of $\mathrm{AD}$, the diagnosis of which is based on clinical features and the exclusion of other causes of dementia. Notwithstanding, the most common research procedures rely on the capability of a single technique to distinguish between patients with diagnosed AD and age-matched healthy controls, or patients affected by other dementing illnesses. Several studies demonstrate that measures of brain functioning (i.e. PET or SPECT) or brain structure (MRI) reveal a quite stable portrait of $\mathrm{AD}$, with a pattern of temporoparietal (association cortex) and mesial temporal hypometabolism-hypoperfusion accompanied by atrophy [1-7].

\section{KARGER}

Fax +4161306 1234 E-Mail karger@karger.ch www.karger.com
(C) 2005 S. Karger AG, Basel $1420-8008 / 05 / 0203-0169 \$ 22.00 / 0$

Accessible online at:

www.karger.com/dem
Prof. Dr. Tomás Ortiz Alonso

Centro MEG Dr. Pérez-Modrego, Facultad de Medicina

Pabellón 8, avda Complutense s\n, Universidad Complutense de Madrid ES-28040 Madrid (Spain)

Tel. +34913942 292, Fax +34913942 294, E-Mail cmeg@ect.ucm.es 
Previous reports performed with isolated techniques, although certainly helpful, failed to establish a definite diagnosis of AD. Recently, a new technique, proton magnetic resonance spectroscopy $\left({ }^{1} \mathrm{H}-\mathrm{MRS}\right)$, has been included in the arsenal of functional brain investigation. ${ }^{1} \mathrm{H}$ MRS is a noninvasive technique which provides in vivo neurochemical information of a spectrum of substances [8]. The typical ${ }^{1} \mathrm{H}-\mathrm{MRS}$ spectrum includes the following peaks: N-acetyl-aspartate (NAA), creatine/phosphocreatine $(\mathrm{Cr})$, choline-containing compounds (Cho), glutamate (Glx) and myoinositol (mI). Among these, NAA has been considered a prominent candidate for AD investigation and diagnosis since it is localized only in neurons and absent in glial tissue in mature brains [9], and thus is a general marker of neural integrity. The brain concentration of NAA is reduced in AD [10] and this reduction correlates with the density of senile plaques and neurofibrillary tangles [11]. However, with the publication of some recent reports which failed to observe a significant decrease in NAA concentrations in mildly demented AD patients [12], the hopes generated by initial studies have only partially come true. The study by Huang et al. [12] and some other studies highlighted the role of increased absolute $\mathrm{mI}$ concentrations or mI/NAA ratios [13-15] in AD. Parnetti et al. [13] reported that mI/NAA ratios differed between controls and demented patients, although no systematic analyses of the sensitivity and specificity of such scores were performed.

Magnetoencephalography (MEG) and magnetic source imaging (MSI) represent another set of noninvasive techniques that combine high temporal and high spatial resolution, allowing detection of focal neural activity. Using simple electromagnetic models, for instance a single focal generator in one brain region, it can be tested how well the data fit this model, and if they fit well, the generator can be positioned with higher precision than with conventional EEG, and the localization can be superimposed onto MR slices [16]. To the best of our knowledge, only three studies have employed MEG to investigate AD by means of spontaneous activity measurements [17-19]. Berendse et al. [17] concluded that absolute power in lowfrequency bands and inter- and intrahemispheric coherence may assist in distinguishing $\mathrm{AD}$ from healthy control subjects. Fernandez et al. [19] published the first reports on low-frequency magnetic activity source localization by means of DD analysis. These studies demonstrated that AD patients exhibited increased DD for delta and theta bands in the temporoparietal regions, and that such increased activity, especially for delta bands, correlated with and predicted the cognitive and functional status of patients. Particularly, Fernandez et al. [19] showed that the combination of functional (i.e. temporoparietal delta DD) and structural measures (i.e. hippocampal volumes) was capable of increasing the sensitivity of AD diagnosis.

The present investigation followed the approach initiated by the above-mentioned study. Namely, a sample of AD patients and controls was subjected to ${ }^{1} \mathrm{H}-\mathrm{MRS}$ and MEG in order to determine the absolute concentrations of NAA, Cr, Cho, and mI, as well as low-frequency DDs. The sensitivity and specificity of each technique for the differentiation between $\mathrm{AD}$ patients and controls were systematically tested by means of logistic regression analysis. In addition, the sensitivity of a combined ${ }^{1} \mathrm{H}-\mathrm{MRS}$ MEG model was tested. It was hypothesized that a combination of functional but highly distinct techniques will improve the diagnosis of $\mathrm{AD}$.

\section{Materials and Methods}

\section{Subjects}

Ten patients (mean age, $65.8 \pm$ SD 7.4 years; 6 females) and 10 controls (mean age $64.4 \pm$ SD 5.4; 8 females) participated in this study. Participants were recruited from the Asociación de Familiares de Enfermos de Alzheimer (AFAL). The clinical diagnosis of AD was ascertained by means of exhaustive medical, neurological, psychiatric and neuropsychological examinations. All patients fulfilled the criteria for probable AD according to the guidelines of the National Institute of Communicative Disorders and Stroke and the AD and Related Disorders Association (NINCDS-ADRDA) [20]. Cognitive status was screened with the Spanish version of the Mini Mental State Examination (MMSE) [21] (maximum score is 35 points). AD patients had MMSE mean scores of 18.6 points (4.8 $\mathrm{SD}$ ), and controls achieved mean scores of 34.2 points (1.03 SD). Consequently, all AD patients were categorized as mildly to moderately demented.

Patients and controls were free of significant medical, neurologic and psychiatric diseases (other than AD), and were not using psychoactive drugs. Prior to the MEG recording, all subjects or their legal representatives signed an informed consent that explained the technical and ethical considerations of the technique. The study was approved by the local Ethics Committee.

\section{${ }^{1} H$-MRS-Collection and Analysis}

MR examinations were performed on a 1.5-tesla clinical MR imager (Signa Horizon Echo Speed v5.8, General Electric Medical System, Milwaukee, Wisc., USA) using the standard quadrature transmit/receive head coil.

Prior to spectroscopy, $\mathrm{T}_{2}$-weighted axial images of the brain covering the parieto-temporo-occipital 'carrefour' region were obtained through a fast spin echo sequence $(\mathrm{TR}=3 \mathrm{~s}$, effective $\mathrm{TE}=$ $96 \mathrm{~ms}$, echo train length $=8$, slice thickness $=4 \mathrm{~mm}$, interslice gap $=1 \mathrm{~mm}$, field of view $(\mathrm{FOV})=24 \times 24 \mathrm{~cm}^{2}$, matrix size $=$ $256 \times 256)$. These images were employed to define the spectroscopic volume of interest (VOI) as shown in figure 1. For each sub- 


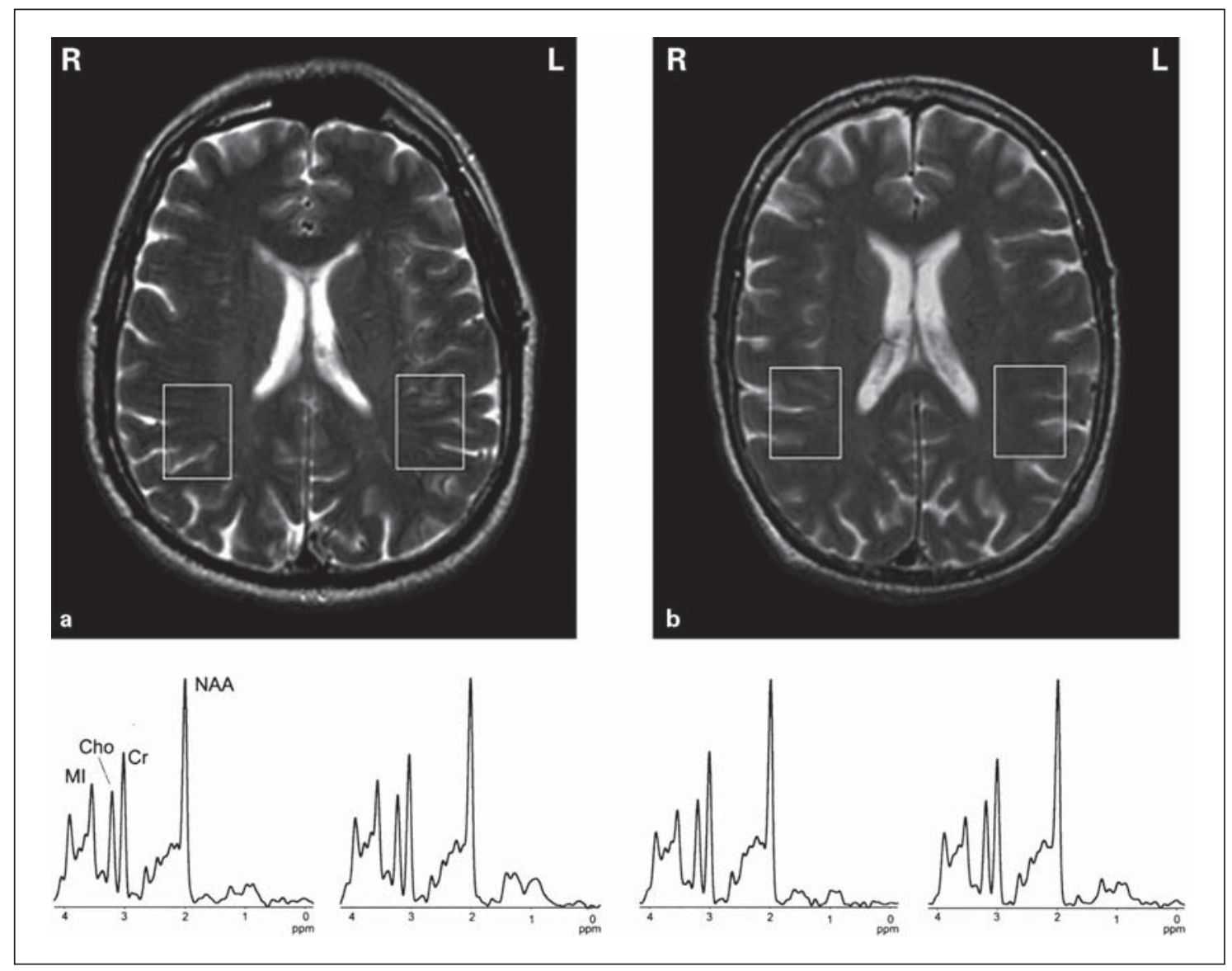

Fig. 1. Axial $T_{2}$-weighted $M R$ images from an $A D$ patient (a), and the corresponding healthy subject employed as control (b). These images show the parietotemporooccipital regions from which localized spectra were acquired. In order to illustrate the kind of spectroscopic results so obtained, the spectra proceeding from each of these spectroscopic voxels are shown under the respective images. $\mathrm{L}=\mathrm{Left} ; \mathrm{R}=$ right.

ject, patient or control, two proton MR spectra from these parietotemporal volumes, one for each hemisphere, were acquired. In addition, 5 individuals of each group were also subjected to spectroscopic acquisitions from volumes in the occipital region of both hemispheres. No significant differences were found in these occipital spectra between hemispheres of the same subject nor between groups. In all cases, spectroscopic acquisitions were performed with the standard probe-p procedure of the GE package (which is based on a double spin echo sequence-PRESS), setting $\mathrm{TR}$ at $3 \mathrm{~s}$ and TE at $35 \mathrm{~ms}$ [22]. Other variables of this GE procedure were opnex $=8$, total_refs $=16$, and total_scans $=64$, which result in an 8 phase-cycling scheme for 16 non-water-suppressed acquisitions ( 2 blocks of 8 averaged scans) and 64 water-suppressed acquisitions ( 8 blocks of 8 averaged scans). The individual raw data files so obtained were analyzed using LCModel software v5.2.2 [23] which automatically performed eddy-current correction of watersuppressed data using the non-water-suppressed lines. LCModel estimates in vivo metabolite concentrations by calculating the linear combination of model metabolite spectra that best fit the in vivo MR spectra.
Our basic set of model metabolite spectra was the one called 'ge_press_te35_95d.basis' which was available from GE Medical Systems in Fremont, Calif. We modified this basic set by including parameterized components for macromolecules and lipids as described elsewhere [24]. This extension of the standard basic set was adopted in order to improve the spectral quantification provided by LCModel, rather than pursuing the estimation of lipids and macromolecules, which were minor or absent contributions in the acquired spectra. With this approach, the metabolite concentrations more reliably estimated by LCModel $(\% \mathrm{SD}<15 \%)$ were those of NAA, total $\mathrm{Cr}$, Cho, and $\mathrm{mI}$. Due to the complications and inconveniences of in vivo $T_{1}$ and $T_{2}$ determinations, we did no attempt to correct relaxation effects. Apart from this, we applied the absolute quantification strategy described by Ernst et al. [25]. Hence, the metabolite concentrations reported herein are those determined by LCModel after water-scaling the individual data set as described in chapter 10 of the LCModel manual. For such a purpose, water concentration as well as the attenuation factor of the unsuppressed water signal were determined for each voxel as already described [25], 2-(trimethyl-silyl) ethanol being employed 
Table 1. Descriptive statistics for metabolite variables

\begin{tabular}{|c|c|c|c|}
\hline & Diagnosis & Mean & $\mathrm{SD}$ \\
\hline \multirow[t]{2}{*}{ LTP_Cho } & $\mathrm{AD}$ & 1.4224 & 0.2704 \\
\hline & control & 1.2695 & 0.1214 \\
\hline \multirow[t]{2}{*}{ RTP_Cho } & $\mathrm{AD}$ & 1.5213 & 2.2692 \\
\hline & control & 1.3337 & 0.1168 \\
\hline \multirow[t]{2}{*}{ LTP_Cr } & $\mathrm{AD}$ & 6.1253 & 0.6614 \\
\hline & control & 5.3284 & 0.3991 \\
\hline \multirow[t]{2}{*}{ RTP_Cr } & $\mathrm{AD}$ & 6.2962 & 0.8836 \\
\hline & control & 5.5153 & 0.5728 \\
\hline \multirow[t]{2}{*}{ LTP_mI } & $\mathrm{AD}$ & 4.7324 & 0.7399 \\
\hline & control & 3.5873 & 0.3383 \\
\hline \multirow[t]{2}{*}{ RTP_mI } & $\mathrm{AD}$ & 4.7247 & 0.8169 \\
\hline & control & 3.7284 & 0.6203 \\
\hline \multirow[t]{2}{*}{ LTP_NAA } & $\mathrm{AD}$ & 7.9620 & 0.6319 \\
\hline & control & 8.1140 & 0.4891 \\
\hline \multirow[t]{2}{*}{ RTP_NAA } & $\mathrm{AD}$ & 8.6922 & 1.2278 \\
\hline & control & 8.0785 & 0.6923 \\
\hline \multirow[t]{2}{*}{ LTP_NAA/Cho } & $\mathrm{AD}$ & 5.7589 & 1.0523 \\
\hline & control & 6.3980 & 0.7531 \\
\hline \multirow[t]{2}{*}{ RTP_NAA/Cho } & $\mathrm{AD}$ & 5.6761 & 0.6732 \\
\hline & control & 6.2025 & 0.8241 \\
\hline \multirow[t]{2}{*}{ LTP_NAA/Cr } & $\mathrm{AD}$ & 1.3144 & 0.1821 \\
\hline & control & 1.4991 & 0.1811 \\
\hline \multirow[t]{2}{*}{ RTP_NAA/Cr } & $\mathrm{AD}$ & 1.3853 & 0.1203 \\
\hline & control & 1.4470 & 0.0866 \\
\hline \multirow[t]{2}{*}{ LTP_mI/Cr } & $\mathrm{AD}$ & 0.7722 & 0.0800 \\
\hline & control & 0.6685 & 0.0829 \\
\hline \multirow[t]{2}{*}{ RTP_mI/Cr } & $\mathrm{AD}$ & 0.7515 & 0.0795 \\
\hline & control & 0.6887 & 0.0758 \\
\hline \multirow[t]{2}{*}{ LTP_mI/NAA } & $\mathrm{AD}$ & 0.5970 & 0.0987 \\
\hline & control & 0.4430 & 0.0471 \\
\hline \multirow[t]{2}{*}{ RTP_mI/NAA } & $\mathrm{AD}$ & 0.5431 & 0.0411 \\
\hline & control & 0.4626 & 0.0451 \\
\hline
\end{tabular}

and placed as external standard according to this reference. This absolute quantification method allows determination of the relative contents of CSF, tissue water and NMR-invisible components in the analyzed voxels, thus making it possible to correct the concentrations determined by LCModel for partial volume effects. As a consequence, the metabolite concentrations reported herein are expressed as moles of metabolite per volume of brain tissue (excluding CSF); that is to say in $c_{\mathrm{bv}}$ in Kreis et al. [26], only lacking the mentioned correction for $\mathrm{T}_{1}$ and $\mathrm{T}_{2}$ effects.

Comparisons between $\mathrm{AD}$ patients and their respective controls were based on the absolute metabolite concentrations. The scores for every metabolite and hemisphere were calculated for the temporoparietal region. Therefore, 16 metabolite variables are included in the statistical analysis: left temporoparietal Cho (LTP_Cho), right temporoparietal Cho (RTP_Cho), left temporoparietal Cr (LTP $\mathrm{Cr}$ ), right temporoparietal $\overline{\mathrm{Cr}}(\mathrm{RTP} \mathrm{Cr})$, left temporoparietal $\mathrm{mI}$ (LTP_mI), right temporoparietal mI (RTP_mI), left temporoparietal NAA (LTP_NAA), right temporoparietal NAA (RTP_NAA), left temporoparietal NAA/Cho quotient (LTP_NAA/Cho), right temporoparietal NAA/Cho quotient (RTP_NAA/Cho), left temporoparietal NAA/Cr quotient (LTP_NAA/Cr), right temporoparietal NAA/Cr quotient (RTP_NAA/Cr), left temporoparietal mI/Cr quotient (LTP_mI/Cr), right temporoparietal $\mathrm{mI} / \mathrm{Cr}$ quotient (RTP $\mathrm{mI} / \mathrm{Cr}$ ), left temporoparietal mI/NAA quotient (LTP_mI/NAA), and right temporoparietal mI/NAA quotient (RTP_mI/NAA). Descriptive statistics of these variables are displayed in table 1.

\section{MEG Data Collection and Analysis}

The MEG was measured using a 148-channel whole-head magnetometer (Magnes ${ }^{\circledR} 2500$ WH,4D Neuroimaging., San Diego, Calif., USA) during a 10-min resting period. Recordings were obtained in supine position, and subjects were asked to stay awake, and to avoid head and eye movement by fixating on a black point on the ceiling. The MEG was recorded with a $678.17-\mathrm{Hz}$ sampling rate, using a band-pass filter of $0.1-200 \mathrm{~Hz}$. Eye movements (EOG) were recorded from four electrodes attached to the left and right outer canthus and above and below the left eye. The electrocardiogram (ECG) was monitored with electrodes attached to the right collarbone and the lowest left rib.

Each 10-min data set was decimated (which consisted of filtering the data in order to respect Nyquist criteria [27], followed by a down-sampling by factor of 16) and band-pass filtered prior to the analysis in the delta $(1.5-4.0 \mathrm{~Hz})$ and theta $(4.0-8.0 \mathrm{~Hz})$ bands. Dipole densities were estimated based on an equivalent current dipole in a homogeneous sphere calculated for each time point. Then, artifact-free time segments of varying lengths were determined by visual inspection. Low-frequency artifacts were typically due to eye movements, identified by EOG. For statistical analysis the total brain volume was divided into five regions, frontal, parietal, prefrontal, temporal (including mesial temporal region) and occipital areas for each hemisphere, and the number of successful dipole fits was determined for each of these regions. The total number of dipoles per second fitting the criteria in the delta and theta bands was determined for each subject and region. In order to avoid the influence of individual variability in the lengths of artifact-free segments, the raw number of dipoles per region was normalized by calculating the 'number of dipoles per second per region' [18, 19, 28]. Single equivalent dipoles were fitted for each time point in the selected epochs.

Considering the main goal of this investigation and following previous studies on LFMA in AD [18, 19], only DD scores (i.e. number of dipoles per second per region) in the temporoparietal areas were utilized for subsequent statistical analysis. Therefore, LFMA DD was represented by 8 variables: left parietal delta DD (LPD_DD), right parietal delta DD (RPD_DD), left temporal delta DD (LTD_DD), right temporal delta DD (RTD_DD), left parietal theta DD (LPT_DD), right parietal theta DD (RPT_DD), left temporal theta DD (LTT_DD), and right temporal theta DD (RTT_DD). Descriptive statistics of these variables are displayed in figure 2. The method was described in detail elsewhere [19, 28].

\section{Statistical Methods}

We developed a series of logistic regression models to predict the diagnosis. First, factors with the highest predictive capability were selected. Then, a value for Alzheimer/control probability was assigned to each patient. The models' performance was assessed by means of classification tables, together with the usual goodness of fit tests.

All the metabolite and DD variables mentioned in the Method section were treated as predictors or independent variables. The outcome or dependent variable was considered to be the correct diagnosis as verified after exhaustive clinical evaluation. The relationship between cognitive (MMSE) evaluations, variations of neu- 
Fig. 2. Mean distribution of parietotemporal DD for delta and theta bands. Y axis shows the number of dipoles per second in every region. Bars in black correspond to $\mathrm{AD}$ group and bars in white correspond to control group.

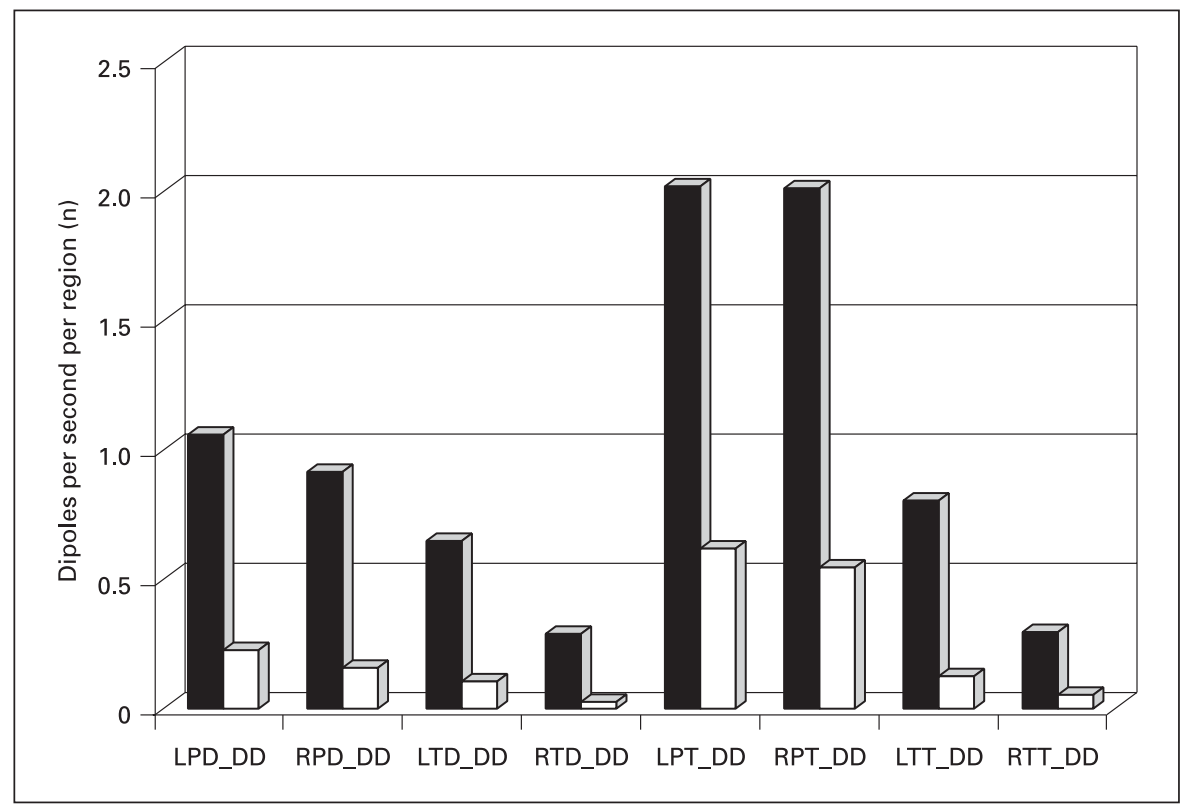

romagnetic activity, and metabolite concentrations were also evaluated. First, a correlation analysis was performed on cognitive, neuromagnetic-regional, and metabolite variables. Then, a linear regression analysis was carried out to define the predictability of the relationship between the neuromagnetic-regional and metabolite variables, and the cognitive scores.

Statistical analyses were performed using the SPSS ${ }^{\circledR}$ statistical package.

\section{Results}

\section{Metabolite and LFMA DD Statistical Analysis}

Prior to selecting the variables, two separate groups of predictor variables were considered: metabolite scores, and LFMA scores (delta and theta bands). The variable selection process began with an univariate analysis for each variable. According to Hosmer and Lemeshow [29], we used a $p$ value of 0.25 for the likelihood ratio test (LRT) as screening criterion to select candidate variables for every multivariate model.

\section{Metabolite Scores}

Following the above-described scheme, univariate analyses selected LTP_mI/NAA and RTP_mI/NAA for multivariate modeling. The coefficients of both variables $(B=-31.05$, and $B=-6.70$, respectively) had the same negative sign: the probability of being a control subject increases when the values of both variables decrease. The linearity in the logit scale for both variables was ascer- tained, and the analysis showed evidence for linearity in both cases. The inclusion of an interaction term did not add any significant improvement. Nevertheless, based on the traditional approach of statistical model building that seeks for the most parsimonious model that still explains the data, an extra step was taken. An attempt to summarize the information contained in both variables was made by creating a new one: mI/NAA_avg $=($ LTP_mI $/$ $\mathrm{NAA}+\mathrm{RTP} \_\mathrm{mI} / \mathrm{NAA} / 2$, which represents an average of the right and left mI/NAA quotients for each patient. A new model containing only mI/NAA_avg was fitted. The estimated coefficient for this variable was $\mathrm{B}=-40.37$, $\mathrm{p}=0.01$ (Wald statistic) and the goodness of fit in -2log likelihood units, 12.6, lower than 16.21, the goodness of fit of the bivariate model. Therefore, the univariate model with mI/NAA_avg substantially improves the bivariate model with LTP_mI/NAA/RTP_mI/NAA. The Nagelkerke $\mathrm{R}^{2}$ goodness of fit statistic (NS) was 0.70 , which represents that about $70 \%$ of the 'variation' in the outcome variable is explained by the logistic regression model. This model was named 'Model 1'.

\section{LFMA DD Scores}

LTD_DD was the factor with the greatest predictive power for focus localization, but all delta and theta DD (LPD_DD, RPD_DD, RTD_DD, LPT_DD, RPT_DD, LTT_DD, and RTT_DD) variables showed a strong association with the outcome after the univariate analyses. Considering that all variables included in the univariate 
Fig. 3. Predominant temporoparietal slow activity, represented by white full squares, in an $\mathrm{AD}$ patient. $\mathrm{L}=\mathrm{Left} ; \mathrm{R}=$ right.

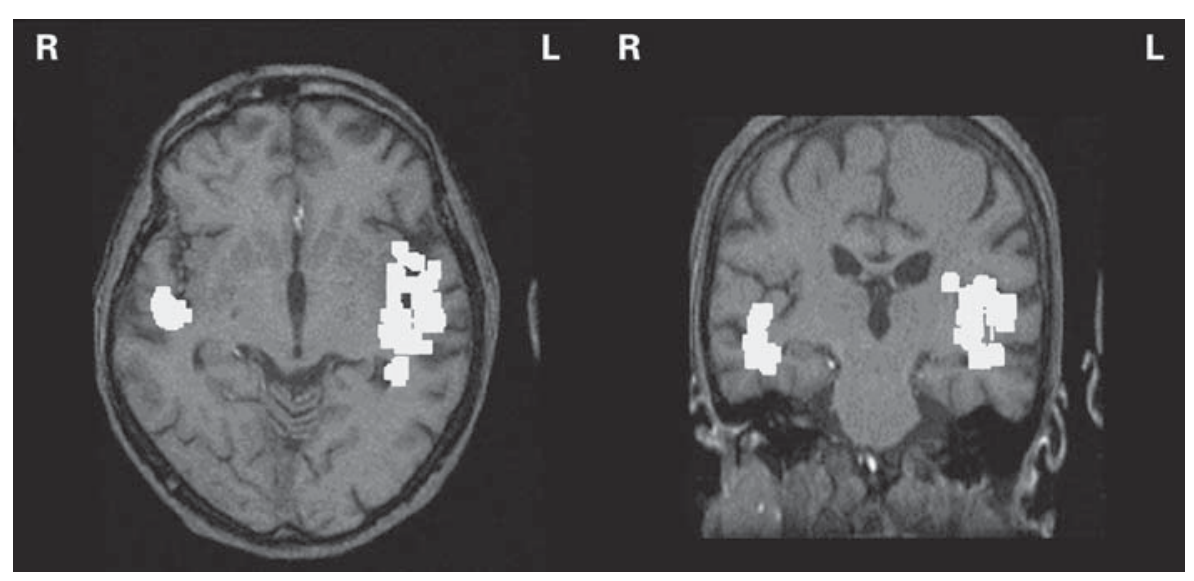

analysis reached a significant value, the information contained in those 8 factors was summarized prior to the multivariate analysis. Two new variables were created: TEPA_D = (LTD_DD + LPD_DD + RPD_DD + RTD DD) $/ 4$; and TEPA_T $=($ LPT_DD + RPT_DD + LTT DD + RTT_DD)/4. The first variable represents the average of the temporoparietal delta activity in both hemispheres for each patient, while the second represents the average of the temporoparietal value for theta activity. Then a multivariate logistic model including the two variables was fitted. Only TEPA_D showed statistical significance $(p<0.05$, LRT). The estimated coefficient for this variable was $B=-7.17, p=0.03$ (Wald statistic) and the goodness of fit in $-2 \log$ likelihood units, 14.92. The NS statistic value was 0.629 . This model was named 'Model 2'.

See figure 3, for an example of DD distribution in an AD patient.

\section{Combined Metabolite and LFMA Analysis}

Comparison between the model's prediction and the observed outcomes is an additional approach for the assessment of model fitting. This information is usually obtained by fixing an arbitrary cutoff for the predicted probability, initially 0.5 , which maximizes the number of correctly classified patients. In our case, when the probability predicted by the model is higher than the cutoff, the subject is considered a control case. If the predicted probability is lower than or equal to the cutoff, the patient is considered an AD case. Then a classification table can be obtained. Tables 2 and 3 display this information for Models 1 and 2, respectively, when the cutoff is 0.5 . The off-diagonal entries of the tables display the number of incorrectly classified patients. Two cases were misclassified by both models. However, the two incorrectly classi-
Table 2. Classification table for Model 1 with cut-off 0.5

\begin{tabular}{llll}
\hline Observed diagnosis & \multicolumn{3}{l}{ Predicted diagnosis, Model 1 } \\
\cline { 2 - 4 } & AD & control & correct \\
\hline AD & 9 & 1 & $90 \%$ \\
Control & 1 & 9 & $90 \%$ \\
& & & overall $90 \%$ \\
\hline
\end{tabular}

The off-diagonal entries of the table display the number of incorrectly classified patients. A control case and an AD patient were misclassified by the model. Outcome is defined as the participant's diagnosis as AD or control defined by the clinical evaluation.

fied patients in table 2 (an AD patient and a control case) are different from those in table 3 ( 2 AD patients). Namely, Model 1 correctly classifies the patients incorrectly classified by Model 2, and vice versa. This strongly suggests a complementary behavior of the models, and advocates the fitting of a final logistic model (Model 3) that comprises selected variables of Model 1 and Model 2: mI/NAA_avg and TEPA_D. The fitted bivariate model has the following coefficients: constant $\mathrm{B}_{0}=31.35$, $\mathrm{B}_{\mathrm{mI} / \mathrm{NAA} \_a v g}=-58.73$, and $\mathrm{B}_{\mathrm{TEPA} \_\mathrm{D}}=-6.75$. The inclusion of an interaction term did not add any significant improvement. The NS statistic value was 0.90 . Also, the $\chi^{2}$ goodness of fit statistic was 2.35 , which, with 8 degrees of freedom yields a $\mathrm{p}$ value of 0.96 , demonstrating a very good fit. Table 4 summarizes the predictive capability of the final logistic model (Model 3). Results indicate that with a cutoff of 0.4 , a $95 \%$ of the cases are correctly classified by Model 3, demonstrating 90\% sensitivity and $100 \%$ specificity. The model predicted a probability value $<0.1$ for the $90 \%$ of $\mathrm{AD}$ cases and a probability value $>0.90$ for the $90 \%$ of control cases. As a consequence, 
even if a very restrictive approach were adopted, and only probability values lower than 0.1 or higher than 0.9 were utilized for patient classification and diagnostic prediction, 18 out of 20 cases $(90 \%)$ would still be correctly classified.

\section{Correlation and Multiple Linear Regression Analysis}

Spearman's correlation coefficients were calculated for the two predictive variables selected by the logistic regression analysis and MMSE scores. Previously, a strong positive correlation was found between the two predictive variables, TEPA_D and mI/NAA_avg $(\mathrm{r}=0.563 ; \mathrm{p}<$ $0.01)$. This strong correlation was further confirmed by the highly significant negative correlations that both variables obtained with MMSE scores: $\mathrm{r}=-0.537 ; \mathrm{p}<0.01$ (TEPA_D), and $\mathrm{r}=-7.82 ; \mathrm{p}<0.001$ (mI/NAA_avg). These correlations indicate that both an increased temporoparietal delta DD and mI/NAA level are associated with lower MMSE scores, and therefore with an impaired cognitive status.

Finally, the capability of TEPA_D and mI/NAA to predict MMSE scores was tested by means of linear regression analysis. Multiple linear regression analysis indicates that a combination of both variables predicts the variability of MMSE $\left(F_{2,19}=14.13 ; p<0.001\right)$. The combination of TEPA_D and mI/NAA explains a $62 \%$ of MMSE variability. Furthermore, the sign of the coefficients of independent variables confirms the tendency evidenced by the correlation analysis: higher scores in both TEPA_D and mI/NAA_avg significantly predict lower scores in MMSE and, as a consequence, a worse mental status of the participants.

\section{Discussion}

The present results confirm our previous hypothesis: a combination of ${ }^{1} \mathrm{H}$-MRS-based and MEG-based scores improves the sensitivity and specificity of $\mathrm{AD}$ diagnosis. Additionally, results concerning temporoparietal delta $\mathrm{DD}$, obtained in a new sample of patients and controls, further support the relevance of this measure in the diagnosis of $\mathrm{AD}$. In a previous study [18], we explained the slowing of AD magnetoelectric activity by the so-called 'cholinergic hypothesis' [30, 31]. Some classical studies $[32,33]$ discovered the association of the cholinergic-dependent ascending reticular system and neocortical EEG activity. Reiekkinen et al. [30] detected a significant correlation between AChE activity of the cerebrospinal fluid (CSF) and the delta power in patients with AD. In au-
Table 3. Classification table for Model 2 with cutoff 0.5

\begin{tabular}{llcc}
\hline \multirow{2}{*}{ Observed diagnosis } & \multicolumn{3}{l}{ Predicted diagnosis, Model 2} \\
\cline { 2 - 4 } & $\mathrm{AD}$ & control & correct \\
\hline AD & 8 & 2 & $80 \%$ \\
Control & 0 & 10 & $\begin{array}{l}100 \% \\
\text { overall } 90 \%\end{array}$ \\
\hline
\end{tabular}

The off-diagonal entries of the table display the number of incorrectly classified patients. Two AD patients were misclassified by the model Outcome is defined as the participant's diagnosis as AD or controls defined by the clinical evaluation.

Table 4. Classification table for Model 3 with cutoff 0.4

\begin{tabular}{llcc}
\hline Observed diagnosis & \multicolumn{3}{l}{ Predicted diagnosis, Model 3} \\
\cline { 2 - 4 } & $\mathrm{AD}$ & control & correct \\
\hline AD & 9 & 1 & $90 \%$ \\
Control & 0 & 10 & $\begin{array}{l}100 \% \\
\text { overall } 95 \%\end{array}$ \\
\hline
\end{tabular}

The off-diagonal entries of the table display the number of incorrectly classified patients. Only one AD patient was misclassified by the model. Outcome is defined as the participant's diagnosis as $\mathrm{AD}$ or control defined by the clinical evaluation.

topsy studies, these authors also observed that patients with the highest EEG delta power had an extremely low cell density in the nucleus basalis of Meynert, and low AChE activity in the cortex. In sum, the most prominent symptoms of $\mathrm{AD}$ appear to be closely related with the slowing of neuroelectric activity, particularly in the temporoparietal regions [34]. Nevertheless, EEG localization reports should be interpreted with care. The most common quantification procedure for EEG is spectral analysis by Fast Fourier Transformation, which depends on a 'recording reference' point. Different references, however, lead to different results. Furthermore, due to the distortion of volume currents by body tissue with varying conductivities, realistic EEG-based source localization is cumbersome and its precision quite limited compared with MEG [16]. This evidence increases the potential value of a stable and MEG-based temporoparietal source of low-frequency activity in AD patients.

A cholinergic deficit is not the only possible explanation for increased delta power and DD. Focal delta activity could be associated with white matter or gray matter 
lesions $[35,36]$. According to the reports of Gloor et al. [35] and Schaul et al. [36], focal polymorphic delta activity is associated with white matter lesions or with a corticothalamic disconnection, while paroxysmal monomorphic delta waves are associated with disturbances of midline structures. Recent investigations demonstrated that not only gray matter but also white matter lesions are present in AD patients' brains [37-39]. In particular, Rose et al. [40], utilizing the novel technique of diffusion tensor imaging, reported a significant reduction in the integrity of AD patients' association white matter tracts. Although the cholinergic hypothesis and the presence of white matter lesions are not mutually exclusive explanations for AD delta activity, the information provided by ${ }^{1}$ H-MRS-based metabolite activity might shed some light on this important issue.

Our results support previous investigations which showed that $\mathrm{mI}$ absolute levels and, particularly, mI/NAA ratios were the most sensitive factors to distinguish mild AD from normal aging [12-15]. As described above, a series of investigations performed by Parnetti et al. [8] pointed to the specific role of mI/NAA. According to their results, $\mathrm{AD}$ patients showed a significant decrease in gray and white matter NAA, and a significant increase in gray matter mI. Nevertheless, only the mI/NAA ratio was capable of classifying ADs and controls without any overlap between groups. Moreover, mI but not NAA showed an inverse linear correlation with MMSE scores. These findings are in good agreement with ours. Then, the questions addressed by these investigations are: (1) Is there an inositol hypothesis for AD? and (2) is there any relationship between increased $\mathrm{mI}$ levels, and the cholinergic reduction or the white matter lesions which might explain the abnormally high delta activity in $\mathrm{AD}$ ? $\mathrm{mI}$ is a well-known candidate for a marker of glial content in brain tissue. Huang et al. [12] proposed that gliosis may cause the increase in $\mathrm{mI}$ observed in $\mathrm{AD}$ patients. If true, this hypothesis would suggest that glial proliferation is a sensitive measure of AD before neuronal loss, as reflected by NAA.
Shonk and Ross [41] previously supported this hypothesis by showing that adults with Down syndrome exhibited a significant elevation of $\mathrm{mI}$ before the onset of dementia, and without any concomitant reduction in the metabolite NAA. On the other hand, cholinergic receptors in the CNS are believed to act in part through the phosphoinositide pathway [42, 43]. Jolles et al. [44] showed a reduced activity of the inositol polyphosphate enzyme phosphatidyinositol kinase and postulated a specific defect in the inositol polyphosphate cascade in $\mathrm{AD}$, to such a degree that cholinergic activity could be affected. Ross et al. [45] summarized this evidence proposing that an altered $\mathrm{mI}$ must have consequences for the enzyme equilibria and the metabolite concentrations in the inositol polyphosphate cascade. Accordingly, altered cholinergic sensitivity could be expected. This notion constitutes a link between the inositol and the cholinergic hypothesis which might explain the strong correlation between mI/NAA levels and delta DDs found in our study, and the improved sensitivity of a combined $\mathrm{mI} / \mathrm{nAA}$-delta DD model to differentiate mild AD from aged controls. In the point of view of Ross et al. [45], 'the cholinergic hypothesis could be considered in one sense, an extension of the inositol hypothesis'.

As a conclusion, this investigation provides additional support to the notion that a combination of complementary techniques significantly improves the diagnosis of AD. Furthermore, results might contribute to the understanding of the biochemical processes underlying the generation of altered neuromagnetic signals in dementia. Future investigations will elucidate if $\mathrm{AD}$ is associated with a specific pattern of MEG frequency distribution that may exceed low-frequency ranges.

\section{Acknowledgement}

To AFAL and Fundación Caja Madrid for their support for the realization of this study.

\section{References}

$>1$ Rapoport S: Positron emission tomography in Alzheimer's disease in relation to disease pathogenesis: a critical review. Cerebrovasc Brain Metabol Rev 1991;3:297-335.

-2 Jagust WJ, Eberling JL, Reed BR, Mathis CA, Budinger TF: Clinical studies of cerebral blood flow in Alzheimer's disease. Ann NY Acad Sci 1997;826:254-262.
3 Kidron D, Black SE, Stanchev P, Buck B, Szalai JP, Parker J, Szekely C, Bronskill MJ: Quantitative MR volumetry in Alzheimer's disease. Topographic markers and the effect of sex and education. Neurology 1997;49:15041512 .

\footnotetext{
-4 Yamaguchi S, Meguro K, Itoh M, Hayasaka C, Shimada M, Yamazaki H, Yamadori A: Decreased cortical glucose metabolism correlates with hippocampal atrophy in Alzheimer's disease as shown by MRI and PET. J Neurol Neurosurg Psychiatry 1997;62:596-600.
} 
5 Jagust WJ, Haan MN, Reed BR, Eberling JL: Brain perfusion imaging predicts survival in Alzheimer's disease. Neurology 1998; 151: 1009-1013.

6 Juottonen K, Laakso MP, Partanen K, Soininen $\mathrm{H}$ : Comparative MR analysis of the entorhinal cortex and hippocampus in diagnosing Alzheimer disease. AJNR 1999;20:139-144.

$\checkmark 7$ Killiany RJ, Gomez-Isla T, Moss M, Kikinis R, Sandor T, Jolesz F: Use of structural magnetic resonance imaging to predict who will get Alzheimer's disease. Ann Neurol 2000;47: 430-439.

8 Parnetti L, Tarducci R, Palumbo B, Pippi M, Mastriforti R, Gobbi G, Pelliccioli GP: In vivo neurochemical characteristics of normal and pathological aging of the brain: role of magnetic resonance spectroscopy. Arch Gerontol Geriatr 1998:suppl 6;393-398.

9 Simmons M, Frondoza CG, Coyle JT: Immunocytochemical localization of $\mathrm{N}$-acetyl-aspartate with monoclonal antibodies. Neuroscience 1991;45:37-45.

-10 Miller B, Moats RA, Shonk T, Ernst T, Woolley S, Ross BD: Alzheimer's disease: depiction of increased cerebral myo-inositol with proton MR spectroscopy. Radiology 1993;187:433437.

11 Klunk WE, Panchalingan K, Moosy J, McLure RJ, Pettegrew JW: N-acetyl-aspartate and other amino acid metabolites in Alzheimer's disease brain: a preliminary proton nuclear magnetic resonance study. Neurology 1992;42: 1578-1585.

-12 Huang W, Alexander GE, Chang L, Shetty HU, Krasuski JS, Rapoport SI, Schapiro MB: Brain metabolite concentration and dementia severity in Alzheimer's disease: a ${ }^{1} \mathrm{H}-\mathrm{MRS}$ study. Neurology 2001;57:626-632.

13 Parnetti L, Tarducci R, Presciutti O, Lowenthal DT, Pippi M, Palumbo B, Gobbi G, Pelliccioli GP, Senin U: Proton magnetic resonance spectroscopy can differentiate Alzheimer's disease from normal aging. Mech Ageing Dev 1997;97:9-14.

14 Rain GS, Mc Connell JR, Waldman A, Grant $\mathrm{D}$, Chaudry M: Brain proton spectroscopy in dementia: an aid to clinical diagnosis. Lancet 1999;353:1063-1064.

-15 Kantarci K, Jack CR, Xu YC, Campeau NG, O'Brien PC, Smith GE, Ivnik RJ, Boewe BF, Kokmen E, Tangalos EG, Petersen RC: Regional metabolic patterns in mild cognitive impairment and Alzheimer's disease: a ${ }^{1} \mathrm{H}-\mathrm{MRS}$ study. Neurology 2000;55:210-217.

16 Elbert T: Neuromagnetism; in Andrä W, Nowak H (eds): Magnetism in Medicine. New York, Wiley, 1998, pp 190-262.

$\checkmark 17$ Berendse HW, Verbunt JPA, Scheltens P, Van Dijk BW, Jonkman EJ: Magnetoencephalographic analysis of cortical activity in Alzheimer's disease: a pilot study. Clin Neurophysiol 2000;111:604-612.
18 Fernández A, Maestú F, Amo C, Gil P, Fehr T, Wienbruch C, Rockstroh B, Elbert T, Ortiz $\mathrm{T}$ : Focal temporo-parietal slow activity in Alzheimer's disease revealed by magnetoencephalography: Biol Psychiat 2002;52:764-770.

19 Fernández A, Arrazola J, Maestú F, Amo C, Gil-Gregorio P, Wienbruch C, Ortiz T: Correlations of hippocampal atrophy and focal low frequency magnetic activity in Alzheimer's disease: a volumetric MRI-MEG study. Am J Neuroradiol 2003;24:481-487.

20 McKhann G, Drachman D, Folstein M: Clinical diagnosis of Alzheimer's disease: report of NINCDS-ADRDA work group under the auspices of Department of Health and Human Services Task Force on Alzheimer's Disease. Neurology 1984:34:939-944.

21 Lobo A, Ezquerra V: El mini-examen cognoscitivo: un test sencillo y práctico para detectar alteraciones intelectivas en pacientes médicos. Actas Luso-Esp Psiquiatría Psicol Méd 1979; 3:189-202.

-22 Webb PG, Sailasuta N, Kohler SJ: Automated single-voxel proton MRS: technical development and multisite verification. Magn Reson Med 1994;31:365-373.

23 Provencher SW: Estimation of metabolite concentrations from localized in vivo proton NMR spectra. Magn Reson Med 1993;30:672679.

24 Seeger U, Klose U, Mader I: Parameterized evaluation of macromolecules and lipids in proton MR spectroscopy of brain diseases. Magn Reson Med 2003;49:19-28.

25 Ernst T, Kreis R, Ross BD: Absolute quantitation of water and metabolites in human brain. I. Compartments and water. Magn Reson Med 1993;b102:1-8.

26 Kreis R, Ernst T, Ross BD: Absolute quantitation of water and metabolites in human brain. II. Metabolite concentrations. Magn Reson Med 1993;b102:9-19.

27 Oppenhein AV, Schafer RW: Digital Signal Processing. Englewood Cliffs, Prentice-Hall, 1974.

28 Fehr T, Kissler J, Moratti S, Wienbruch C, Rockstroh B, Elbert T: Source distribution of neuromagnetic slow waves and MEG-delta activity in schizophrenic patients. Biol Psychiatry 2001;50:108-116.

29 Hosmer D, Lemeshow S: Applied Logistic Regression. New York, Wiley 1989.

30 Riekkinen P, Buzsaki G, Riekkinen P, Soininen H, Partanen J: The cholinergic system and EEG slow waves. Electroencephalog Clin Neurophysiol 1991;78:89-96.

31 Dringenberg HC: Alzheimer's disease: More than a 'cholinergic disorder' - evidence that cholinergic-monoaminergic interactions contribute to EEG slowing and dementia. Behav Brain Res 2000;115:235-249.

32 Longo VG: Effects of scopolamine and atropine on electroencephalographic and behavioral reactions due to hypotalamic stimulation. J Pharmacol 1966;116:198-208.
33 Shute CCD, Lewis PR: The ascending cholinergic reticular systems: neocortical, olfactory and subcortical projections. Brain 1967;90: 497-520.

>34 Dierks T, Jelic V, Pascual-Marqui RD, Wahlund LO, Julin P, Linden DEJ, et al: Spatial pattern of cerebral glucose metabolism (PET) correlates with localization of intracrebral EEG-generators in Alzheimer disease. Clin Neurophysiol 2000;111:1817-1824.

$>35$ Gloor P, Ball G, Schaul N: Brain lesions that produce delta waves in the EEG. Neurology 1977;27:326-333.

36 Schaul N, Gloor P, Ball G, Gotman J: The EEG in deep midline lesions. Neurology 1981;31: 157-167.

37 Hirono N, Kitagaki H, Kazui H, Hashimoto M, Mori E: Impact of white matter changes on clinical manifestation of Alzheimer's disease: a quantitative study. Stroke 2000;31:21822188.

38 Bozzali M, Falini A, Franceschi M, Cercignani M, Zuffi M, Scotti G, Comi G, Filippi M: White matter damage in Alzheimer's disease assessed in vivo using diffusion tensor magnetic resonance imaging. J Neurol Neurosurg Psychiatry 2002;72:742-746.

39 Watanabe T, Akiguchi I, Yagi H, Onishi K, Kawasaki T, Shiino A, Inubushi T: Proton magnetic resonance spectroscopy and white matter hyperintensities on magnetic resonance imaging in patients with Alzheimer's disease. Ann NY Acad Sci 2002;977:423-429.

40 Rose SE, Chen F, Chalk JB, Zelaya FO, Strugnell WE, Benson M, Semple J, Doddrell DM: Loss of connectivity in Alzheimer's disease: an evaluation of white matter tract integrity with colour coded MR diffusion tensor imaging. J Neurol Neurosurg Psychiatry 2000;69: 528-530.

41 Shonk T, Ross BD: Role of increased cerebral myo-inositol in the dementia of Down syndrome. Magn Res Med 1995;33:858-861.

$>42$ Honchar MP, Vogler GP, Gish BG, Sherman WR: Evidence that phosphoinositide metabolism in rat cerebral cortex stimulated by pilocarpine, physostigmine and pargyline in vivo is not changed by chronic lithium treatment. Neurochemistry 1990;55:1521-1525.

43 Kennedy ED, Chaliss JRA, Ragan CL, Nahorski SF: Reduced inositol polyphosphate accumulation and inositol supply induced by lithium in stimulated cerebral cortex slices. Biochem J 1990;267:781-786.

44 Jolles J, Bothmer J, Markerink M, Ravid R: Phosphatidylinositol kinase is reduced in Alzheimer's disease. J Neurochem 1992;58:23262329.

45 Ross BD, Bluml S, Cowan R, Danielsen E, Farrow N, Gruetter R: In vivo magnetic resonance spectroscopy of human brain: the biophysical basis of dementia. Biophys Chem 1997;68: 161-172. 